\title{
PERFIL CLÍNICO DE PACIENTES DE UM PROGRAMA DE REABILITAÇÃO CARDÍACA QUE APRESENTAM RISCO DE TER DIABETES
}

Beatriz Espanhol Garcia, Laís Manata Vanzella, Ana Paula Soares dos Santos, Elaine Aparecida de Oliveira, Carolina Takahashi, Thais Cabral Almeida, Paula Rapchan dos Santos, Aline Ferreira Lima Gonçalves, Ana Alice Soares dos Santos, Thais Roque Giacon, Natália Turri da Silva, Aline Fernanda Barbosa Bernardo, Luiz Carlos Marques Vanderlei

Universidade Estadual Paulista Júlio de Mesquita Filho - UNESP. Departamento de Fisioterapia, Presidente Prudente SP. Pro- Reitoria de Extensão Universitária - PROEX.

\section{RESUMO}

O Diabetes Mellitus (DM) é uma doença de caráter crônico e que exerce influência negativa sobre os componentes do sistema cardiovascular. O objetivo do estudo foi avaliar o risco de desenvolver diabetes em cardiopatas participantes de um programa de reabilitação cardíaca. 20 pacientes responderam o questionário Finnish Diabetes Risk Score revelando o risco de desenvolvimento da DM em 10 anos. O questionário avalia a idade, prática de atividade física (AF), consumo diário de frutas e/ou verduras, uso de anti-hipertensivos, história de glicose sanguínea alta e história familiar de diabetes. Apenas $5 \%$ dos pacientes foram classificadas como baixo risco, sendo que a maior porcentagem se encontra na faixa de moderado a alto risco, e $40 \%$ apresentaram risco alto a muito alto de desenvolver DM. Conclui-se que o risco de indivíduos cardiopatas apresentarem DM ao longo da vida é muito alto, devido à presença dos fatores de risco (FR) associados à doença. Palavras-chave: diabetes mellitus; cardiopatias; cardiopatas; complicações do diabetes; fatores de risco.

\section{CLINICAL PROFILE OF PATIENTS OF A CARDIAC REHABILITATION PROGRAM THAT HAVE RISK OF DIABETES}

\begin{abstract}
Diabetes Mellitus is a chronic disease and that exerts negative influence on the components of the cardiovascular system. The aim of the study was to evaluate the risk of developing diabetes in participants in a cardiac rehabilitation program. 20 patients completed the questionnaire Finnish Diabetes Risk Score reveals that the risk of developing the disease in 10 years. The questionnaire assesses age, physical activity, daily consumption of fruits and / or vegetables, use of antihypertensive drugs, history of high blood glucose and family history of diabetes. Only $5 \%$ of patients were classified as low risk, with the highest percentage is in the moderate to high risk, and $40 \%$ have high or a very high risk of developing diabetes mellitus. It is concluded that the risk of cardiac patients experiencing diabetes mellitus throughout life is very high due to the presence of risk factors associated with the disease.
\end{abstract}

Keywords: diabetes mellitus; heart diseases; cardiacs; diabetes complication; risck factors. 


\section{INTRODUÇÃO}

A Diabetes Mellitus (DM), é uma doença de caráter crônico e evolutivo, envolvendo distúrbios no metabolismo das proteínas e lipídios, e pela deficiência relativa ou absoluta da insulina que leva o paciente a apresentar hiperglicemia ${ }^{1}$. Em 2002, estimava na população mundial a presença de 173 milhões de diabéticos, sendo a projeção para 2030 de 366 milhões. Esse aumento estaria associado ao crescimento e envelhecimento populacional, aos maus hábitos de vida da população, como crescente consumo de gorduras, sedentarismo que leva a obesidade, e em virtude da maior sobrevida de pacientes com $\mathrm{DM}^{2}$.

A DM é a quinta causa de morte no mundial $^{3,4}$, entretanto, os indivíduos com DM na maioria das vezes não morrem de causas exclusivamente relacionadas á patologia, mas sim de complicações cardiovasculares e renais ${ }^{5}$, sendo a doença arterial coronariana (DAC) a principal causa de morbidade e mortalidade nesses pacientes ${ }^{6,7}$. Estima-se que o DM aumente o risco de duas a quatro vezes da DAC, e de síndromes coronarianas agudas (SCA) cuja incidência em diabéticos chega a 20\% em 7 anos, contra 3,5\% em nãodiabéticos ${ }^{8}$. Ainda, pacientes diabéticos são mais propensos ao infarto agudo do miocárdio (IAM) e insuficiência cardíaca e estão em maior risco de morrer depois de um evento cardíaco agudo ${ }^{9}$.

Além dos fatores genéticos, a obesidade e o sedentarismo são os principais determinantes para o aparecimento de $\mathrm{DM}^{10}$. Entretanto, vários estudos têm mostrado diminuição da incidência do DM por meio de mudança de estilo de vida e redução do peso corporal $^{11}$.

Mesmo em indivíduos inseridos em programas de reabilitação cardíaca, a presença de fatores de risco (FR) cardiovasculares, inclusive do DM, também é elevada ${ }^{12}$. No entanto, estudos referentes ao risco que cardiopatas não diabéticos têm de desenvolver DM no futuro ainda não são encontrados, e detectar a possível presença desse FR é de extrema importância, na tentativa de controlar e reduzir a gravidade dessa doença ${ }^{13}$. O objetivo do presente estudo é avaliar o risco de aparecimento da DM em pacientes cardiopatas não diabéticos participantes de um programa de reabilitação cardíaca.

\section{MÉTODOS}

\section{Amostra}

O estudo tem caráter epidemiológico de corte do tipo transversal e foram avaliados 20 pacientes frequentadores do setor de Reabilitação Cardíaca do Centro de Estudos e de Atendimentos em Fisioterapia e Reabilitação (CEAFIR), sendo a maioria do 
gênero masculino (13 homens) com média de idade de $66,33 \pm 8,45$ anos.

Não foram incluídos no estudo pacientes que já apresentavam DM, os que se recusaram a colaborar com a coleta de dados e aqueles que não compreenderam os questionários. O estudo foi aprovado pelo Comitê de Ética em Pesquisa da FCT/UNESP (CEP 2013/331954).

\section{Coleta de Dados}

Para realizar a coleta de dados, uma equipe treinada visitou os pacientes durante a sessão de reabilitação cardíaca. Foi aplicado um questionário da forma de entrevista e posteriormente, mensurados a massa corporal (balança Welmy, Brasil), estatura (estadiômetro Sanny, Brasil) e calculado o índice de massa corporal (IMC) (peso/ altura $^{2}$ ) de cada indivíduo em posição ortostática e vestindo roupas leves. A circunferência abdominal (CA) (fita métrica Sanny, Brasil) foi mensurada na região da cicatriz umbilical por baixo da blusa.

O questionário aplicado Finnish Diabetes Risk Score (FINDRISC) foi desenvolvido e validado ${ }^{14}$ na Finlândia e por meio de uma pontuação aponta o risco para desenvolver a patologia nos próximos 10 anos, por ser de fácil entendimento e baixo custo, esse questionário vem sendo corriqueiramente utilizado no Brasil ${ }^{15}$.

Composto por oito itens, o questionário investiga dados como a idade, prática de atividade física, consumo diário de frutas e/ou verduras, uso de antihipertensivos, história de glicose sanguínea alta, história familiar de diabetes, entre outros. A pontuação máxima é de 28 pontos, classificando os indivíduos nos níveis de risco: baixo ( $<7$ pontos); levemente moderado (entre 7 e 11 pontos); moderado (entre 12 e 14 pontos); alto (entre 15 e 20 pontos); e muito alto (mais de 20 pontos) ${ }^{14}$.

\section{Análise Estatística}

Para análise dos dados foi realizada a estatística descritiva e os resultados foram apresentados os valores de média, desviopadrões, números absolutos e percentuais.

\section{RESULTADOS}

A média do IMC dos pacientes avaliados foi de $29,30 \pm 6,17$, indicando um sobrepeso, conforme mostra a tabela 1. 
Tabela 1. Variáveis antropométricas da população apresentadas em média e desvio-padrão

\begin{tabular}{cc}
\hline \hline Variável & Valores \\
\hline Idade (anos) & $66,33 \pm 8,45$ \\
Peso $(\mathrm{Kg})$ & $79,07 \pm 6,92$ \\
Altura $(\mathrm{m})$ & $1,64 \pm 0,22$ \\
IMC $\left(\mathrm{Kg} / \mathrm{m}^{2}\right)$ & $29,30 \pm 6,17$ \\
$\mathrm{CA}(\mathrm{cm})$ & $94,95 \pm 8,45$. \\
\hline
\end{tabular}

$\mathrm{Kg}=$ kilogramas; $\mathrm{m}=$ metros; $\mathrm{CA}=$ cincunferência abdominal; $\mathrm{cm}=$ centímetros; $\mathrm{IMC}=$ índice de massa corpórea; $\mathrm{Kg} / \mathrm{m}^{2}=$ kilogramas/metros ${ }^{2}$

Os resultados em relação aos níveis de risco para o desenvolvimento de DM demonstraram que apenas $5 \%$ da população estudada foram classificadas como baixo risco e $40 \%$ dos cardiopatas apresentaram risco alto a muito alto de desenvolver DM, conforme mostra a tabela 2.

Tabela 2. Distribuição da população conforme os níveis de risco para desenvolver diabetes melitus

\begin{tabular}{lll}
\hline \hline Nível de risco & $\mathbf{n}$ & $\%$ \\
\hline Risco baixo & 1 & 5 \\
Risco pouco elevado & 5 & 25 \\
Risco moderado & 6 & 30 \\
Risco alto & 6 & 30 \\
Risco muito alto & 2 & 10 \\
\hline \hline
\end{tabular}

Para análise das variáveis expressas em percentuais, esses intervalos foram categorizados como de risco moderado $(n=11)$ e risco alto $(n=8)$. O grupo de risco baixo apresentou apenas um indivíduo, sendo este excluído desta análise. O resultado das variáveis constituintes do questionário segue na tabela 3. 
Tabela 3. Análise das variáveis constituintes do questionário sobre o risco de ter diabetes.

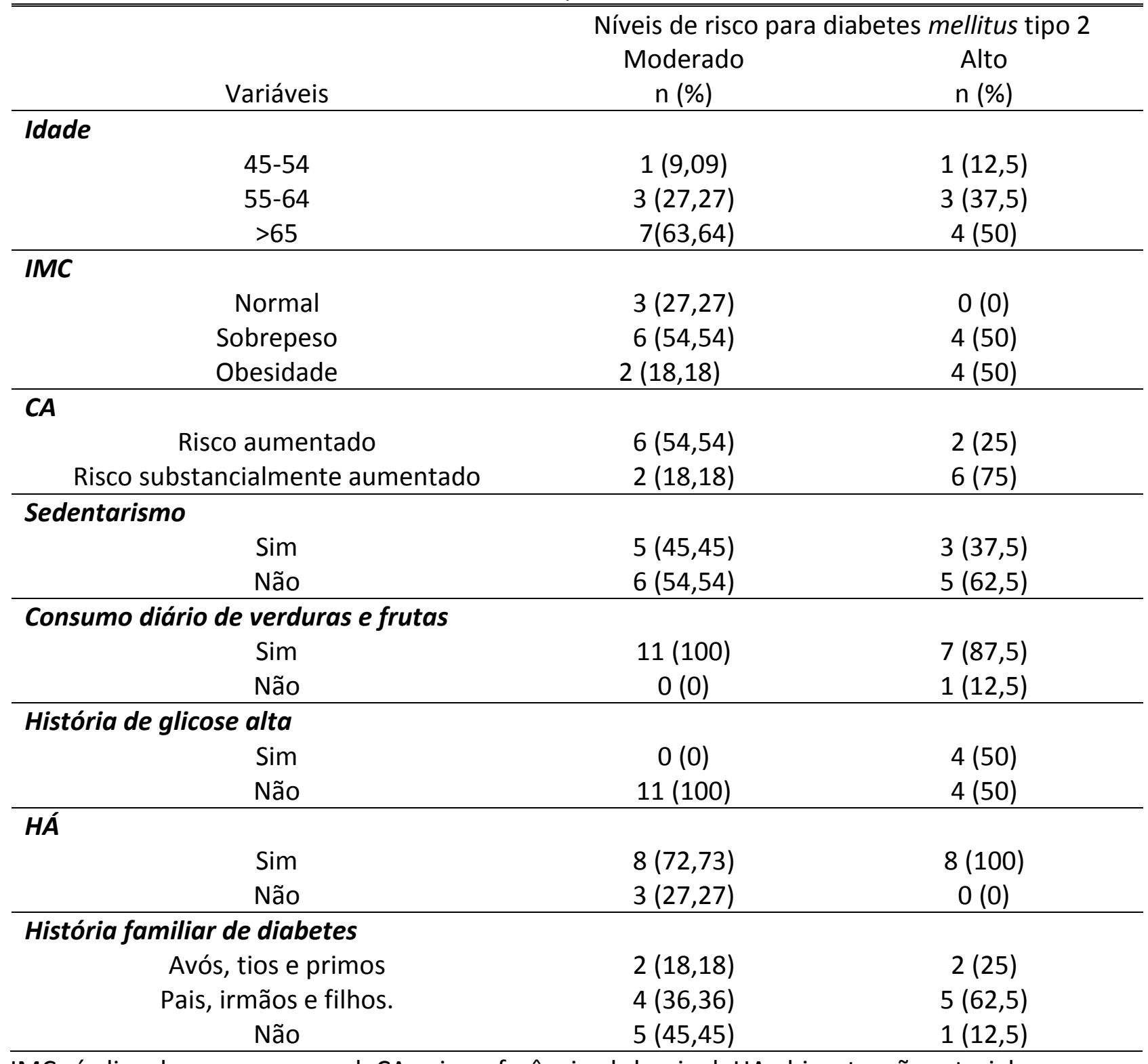

$\mathrm{IMC}=$ índice de massa corporal; $\mathrm{CA}=$ circunferência abdominal; $\mathrm{HA}=$ hipertensão arterial

\section{DISCUSSÃO}

Nesta pesquisa, os indivíduos apresentaram risco para desenvolver DM tipo 2 nos próximos 10 anos, sendo esse risco moderado ou alto para a maioria dos entrevistados. Considerando os FR associados que levam ao risco moderado e/ou alto de ter DM, os mais prevalentes foram relacionados à idade, excesso de peso,
CA aumentada, HA e histórico familiar de DM.

A DM está associação com a presença de outros FR, Marinho et a encontraram como fatores mais prevalentes a CA aumentada $(84,0 \%)$, seguido do sedentarismo $(83,3 \%)$ e do excesso de peso $(57,9 \%)$, e ainda, $98,8 \%$ apresentaram algum nível de risco para DM tipo $2^{16}$. 
Canavan RJMB et al relataram que pode haver cerca de 113 milhões de pessoas com DM com idade acima de 45 anos $^{17}$. Nossos achados evidenciam que quanto maior foi a idade, maior o risco para desenvolver DM em categorias de risco moderado e alto. Sartorelli e Franco citam que entre os principais $F R$ para o DM na população brasileira está a obesidade, o histórico familiar e o envelhecimento ${ }^{18}$. O envelhecimento pode levar á vulnerabilidade de processos patológicos, pois o com o avançar da idade associado a múltiplos fatores, o indivíduo pode apresentar diversas doenças, entre elas estão as cardiovasculares e endócrinas ${ }^{19}$.

O excesso de peso pode está associado ao maior risco de ter DM, uma vez os indivíduos pertencentes ao grupo de alto risco apresentam valores de IMC elevado, sendo $50 \%$ classificados com sobrepeso e $50 \%$ obesos. Pelegrini et al também constataram que o excesso de peso está positivamente associado à DM, independentemente do sexo, idade, escolaridade, atividade física habitual e $\mathrm{HA}^{20}$. A redução do peso é efetiva em reduzir a incidência de DM, como mostrou o Finnish Diabetes Prevention Study (DPS) ${ }^{21}$ no qual uma redução de $3 \mathrm{~kg}$ a $4 \mathrm{~kg}$ diminuiu a incidência de DM em $58 \%{ }^{22}$. O sobrepeso pode causar alterações que levam o organismo apresentar uma resistência á insulina ${ }^{23}$.

Em relação à $C A$, no grupo de risco moderado $18,18 \%$ foram classificadas com risco substancialmente aumentado, enquanto que no grupo do risco alto esse índice se elevou para $75 \%$ dos indivíduos. Estes dados evidenciaram que a presença de obesidade andróide pode ser um risco para doenças como o DM e diversas cardiopatias $^{24}$, uma vez que esse acúmulo de gordura abdominal é prejudicial ao sistema cardiovascular.

A prática de atividade física (AF) é fundamental no tratamento de DM, pois reduzem a necessidade de antidiabéticos orais, diminuem a resistência à insulina e contribuem para um melhor controle glicêmico, reduzindo o risco de complicações ${ }^{25}$. Em nosso estudo $45,45 \%$ dos entrevistados do grupo de risco moderados foram classificados como sedentários, enquanto de no grupo de risco alto rico esse dado foi de $37,5 \%$. O sedentarismo foi muito maior no estudo de Marinho et al, onde $83,3 \%$ da amostra não praticava $A F$ regularmente ${ }^{16}$. Esta diferença entre os achados e estudos se deve ao fato de que os nossos entrevistados frequentam um programa de AF no mínimo três vezes por semana durante uma hora, entretanto o questionário classifica os indivíduos como 
ativos aqueles que realizam AF no mínimo 30 minutos por dia ou quatro horas na semana.

Quanto à dieta, a maioria das pessoas entrevistadas relatou consumir frutas e verduras diariamente, um fator positivo visto que estudos epidemiológicos têm sugerido que uma dieta rica em energia, gorduras saturadas, colesterol e sal favorecem o aparecimento de doenças cardiovasculares $^{26}$. Ainda, nenhum individuo do grupo de risco moderado, apresentou história de glicose alta em exames de rotina, entretanto a glicemia elevada esteve presente em $50 \%$ dos indivíduos do grupo de risco alto. Dessa maneira, esses indivíduos devem fazer o uso de medicamentos para controle da DM, ou ainda, pequenas mudanças nos comportamentos de vida diária.

Estudos mostraram que em diabéticos, a HA é duas vezes mais freqüente que na população em geral $^{27}$ e evidenciaram que indivíduos com história familiar de primeiro grau de DM tipo 2 apresentaram alto risco de desenvolver a doença ${ }^{28}$. No presente estudo, $54,54 \%$ dos indivíduos do grupo de risco moderado apresentaram história familiar de DM e no grupo de risco alto este dado subiu para $87,5 \%$, demonstrando que fatores genéticos influenciam e aumentam o risco desenvolvimento da DM.
Em relação às limitações do estudo, a amostra foi composta por um número relativamente reduzido de participantes, podendo fazer com que os nossos resultados possam apenas ser aplicados a grupos semelhantes aos usados neste estudo.

Este estudo possui implicações clínicas positivas para os profissionais da área da saúde, visto que identificar o risco de desenvolver diabetes em indivíduos cardiopatas é importante por ser um fator de risco cardiovascular. E ao identificar o risco, torna-se possível a adoção de medidas preventivas para evitar o aparecimento da doença e as possíveis complicações a elas inerentes.

\section{CONCLUSÃO}

A partir dos achados, conclui-se que o risco de indivíduos cardiopatas apresentarem diabetes melitus ao longo da vida é muito alto, devido à presença dos fatores associados à doença, sendo mais prevalentes a idade, excesso de peso, circunferência abdominal e histórico familiar.

\section{REFERÊNCIAS}

1. World Health Organization. Diabetes. 2012; (312).

2. Sociedade Brasileira de Diabetes. Diretrizes da Sociedade Brasileira de Diabetes: 2013-2014. AC Farmacêutica, 2014.

3. Roglic $\mathrm{G}$, Unwin $\mathrm{N}$, Bennett $\mathrm{PH}$, Mathers C, Tuomilehto J, Nag S, et al. The 
burden of mortality attributable to diabetes: realistic estimates for the year 2000. Diabetes Care. 2005; 28:(9)2130-5. DOI: http://dx.doi.org/10.2337/diacare.28.9.2130.

4. Allgot $B$, Gan $D$, King $H$, Lefèbvre $P$, Mbanya $C J$, Silink $M$, et al. Diabetes Atlas. International Diabetes Federation. 2003.

5. Morrish NJ, Wang SL, Stevens LK, Fuller $\mathrm{JH}$, Keen $\mathrm{H}$ : Mortality and causes of death in the WHO Multinational Study of Vascular Disease in Diabetes. Diabetologia 2001; 44 (2): 14-21. DOI: http://dx.doi.org/10.1007/PL00002934.

6. Goldberg RB. Cardiovascular disease in diabetic patients. Med Clin North Am. 2000; 84:81-93. DOI: http://dx.doi.org/10.1016/S00257125(05)70208-X.

7. Garcia MJ,McNamara PM, Gordon T, Kannel WB. Morbidity and mortality in diabetics in the Framingham population: sixteen year follow-up study. Diabetes. 1974; 23:105-11.

DOI:

http://dx.doi.org/10.2337/diab.23.2.105.

8. Haffner SM, Lehto S, Rönnemaa T, Pyörälä K, Laakso M. Mortality from coronary heart disease in subjects with type 2 diabetes and in nondiabetic subjects with and without prior myocardial infarction. N Engl J Med 1998;339(4):229-34. DOI: http://dx.doi.org/10.1056/NEJM1998072333 $\underline{90404}$.

9. Herlitz J, Malmberg K, Karlson BW, Ryden L, Hjalmarson A. Mortality and morbidity during a five-year follow-up of diabetics with myocardial infarction. Acta Med Scand. 1998; 224:31-38. DOI: http://dx.doi.org/10.1111/j.09546820.1988.tb16735.x.

10. Wild S, Roglic G, Green A, Sicree R, King $H$. Global prevalence of diabetes: estimates for the year 2000 and projections for 2030. Diabetes Care. 2004;27(5):1047-53. DOI:

http://dx.doi.org/10.2337/diacare.27.5.1047.

11. Diabetes Prevention Program Research Group, Knowler WC, Fowler SE, Hamman RF, Christophi CA, Hoffman HJ, et al. 10-year follow-up of diabetes incidence and weight loss in the Diabetes Prevention Program Outcomes Study. Lancet. 2009; 14;374(9702):1677-86.

12. Bernardo AFB, Fernandes RA, Silva AKF, Valenti VE, Pastre CM, Vanderlei LCM. Influence of risk behavior aggregation in different categories of physical activity on the occurrence of cardiovascular risk factors. International Archives of Medicine. 2013; 6:26. DOI: http://dx.doi.org/ 10.1186/17557682-6-26.

13. Lee TC, Hanlon JG, Ben-David J, Booth GL, Cantor WJ, Connelly PW et al. Risk Factors for Cardiovascular Disease in Homeless Adults. Circulation 2005; May (24): 2629-35. DOI: http://dx.doi.org/ 10.1161/ CIRCULATIONAHA.104.510826.

14. Saaristo T, Etu-Seppala L. Prevención de la diabetes y sus complicaciones: objetivos clave en Finlandia. Diabetes Voice. 2006;51(4):14-7.

15. Bittencourt A, Vinholes DB. Assessing the risk for type 2 diabetes mellitus in bank employees from the city of Tubarao, Santa Catarina state, Brazil. Sci Med. 2013;23(2):82.

16. Marinho PBN, Vasconcelos $\mathrm{ACH}$, Alencar GPMA, Almeida CP, Damasceno CMM. Risco para diabetes mellitus tipo 2 e fatores associados. Acta paul. enferm. 2013; 26(6):569-74.

DOI: http://dx.doi.org/10.1590/S010321002013000600010.

17. Canavan RJMB. Diabetes and nondiabetes related lower extremity amputation incidence before and after the 
introduction of better organized diabetes foot care: contínuos longitudinal monitoring using a standart method. Diabetes Care. 2008; 31 (3): 459-63. DOI: http://dx.doi.org/ 10.2337/dc07-1159.

18. Sartorelli DS, Franco LJ. Tendências do diabetes mellitus no Brasil: o papel da transição nutricional. Cad Saude Publica.2003,19(1): 529-36.

19. Kusumota L, Rodrigues RAP, Marques S. Idosos com insuficiência renal crônica: alterações do estado de saúde. Rev. Latinoam. enfermagem 2004 Maio-Jun; 12 (3): 52532.

20. Pelegrini A, Coqueiro SR, Petroski LE, Benedetti BRT. Diabetes mellitus autoreferido e sua associação com excesso de peso em idosos. Rev. bras. cineantropom. desempenho hum. 2011; 13 (6): 442-47. DOI: http://dx.doi.org/10.5007/1980-

0037.2011v13n6p442.

21. Diabetes Prevention Program Research Group. Reduction of the incidence of type 2 diabetes with life style intervention or metformin. NEngl J Med. 2002; 346(6):393-403. DOI: http://dx.doi.org/10.1056/NEJMoa012512.

22. Tuomilehto J, Lindstrom J, Eriksson JG, Valle TT, Hamalainen $H$, Hanne-Parikka $P$ et al.; for the Finnish Diabetes Prevention Program. Prevention of type 2 diabetes mellitus by changes in life style among subjects with impaired glucose tolerance. $\mathrm{N}$ Engl J Med. 2001; 344 (18):1343-50.

23. Pereira LO, Franscischi RP, Junior AHL. Obesidade: Hábitos nutricionais, sedentarismo e resistência á insulina. Arq Bras Endocrinol Metab 2003; 47/2:111-127).

24. Ortiz ACM, Zanetti LM. Levantamento dos fatores de risco para diabetes mellitus tipo 2 em uma instituição de ensino superior. Rev. Latino-Am. Enfermagem. 2001;9 (3):58-
63. DOI: http://dx.doi.org/10.1590/S010411692001000300009 .

25. Ford ES, Herman WH. Leisure - time physical activity patterns in the U.S. diabetic population. Diabetes Care 1995;18:27-33. DOI:

http://dx.doi.org/10.2337/diacare.18.1.27.

26. Leeds AR. Glycemic index and heart disease. Am J Clin Nutr, 2002; 76(1):286-289.

27. HDS - Hypertension in Diabetes Study Group. HDS 1: Prevalence of hypertension in newly presenting type 2 diabetic patients and association with risc factors for cardiovascular and diabetic complications. Journal of Hypertension, London, 1993; 11(3):309-17.

28. Beck-Nielsen H, Groop LC. Metabolic and genetic characterization of prediabetic states. Sequence of events leading to noninsulin-dependent diabetes mellitus. J Clin Invest. 1994;94(5):1714-721. DOI: http://dx.doi.org/10.1172/JCl117518.

Recebido para publicação em 12/08/2014 Revisado em 27/08/2014

Aceito em 01/09/2014 